\title{
Synthetic vision for lunar and planetary landing vehicles
}

\author{
Steven P. Williams ${ }^{a}$, Jarvis (Trey) J. Arthur, III ${ }^{a}$, Kevin J. Shelton ${ }^{a}$, Lawrence J. Prinzel, III ${ }^{a}$ \\ and Dr. R. Michael Norman ${ }^{b}$ \\ ${ }^{a}$ NASA Langley Research Center, Hampton, VA \\ ${ }^{b}$ Boeing Phantom Works, Hampton, VA
}

\begin{abstract}
The Crew Vehicle Interface (CVI) group of the Integrated Intelligent Flight Deck Technologies (IIFDT) has done extensive research in the area of Synthetic Vision (SV), and has shown that SV technology can substantially enhance flight crew situation awareness, reduce pilot workload, promote flight path control precision and improve aviation safety. SV technology is being extended to evaluate its utility for lunar and planetary exploration vehicles. SV may hold significant potential for many lunar and planetary missions since the SV presentation provides a computer-generated view of the terrain and other significant environment characteristics independent of the outside visibility conditions, window locations, or vehicle attributes. SV allows unconstrained control of the computer-generated scene lighting, terrain coloring, and virtual camera angles which may provide invaluable visual cues to pilots/astronauts and in addition, important vehicle state information may be conformally displayed on the view such as forward and down velocities, altitude, and fuel remaining to enhance trajectory control and vehicle system status. This paper discusses preliminary SV concepts for tactical and strategic displays for a lunar landing vehicle. The technical challenges and potential solutions to SV applications for the lunar landing mission are explored, including the requirements for high resolution terrain lunar maps and an accurate position and orientation of the vehicle that is essential in providing lunar Synthetic Vision System (SVS) cockpit displays. The paper also discusses the technical challenge of creating an accurate synthetic terrain portrayal using an ellipsoid lunar digital elevation model which eliminates projection errors and can be efficiently rendered in real-time.
\end{abstract}

Keywords: Synthetic Vision, Cockpit, Moon, Spacecraft, Modeling, Simulation

\section{INTRODUCTION}

Synthetic Vision System (SVS) emerged from aviation-specific research at NASA and elsewhere to the point where SVS display systems are now available for general aviation and commercial aircraft. SVS is a computergenerated image of the external scene topography from the perspective of the flight deck that is derived from aircraft attitude, high-precision navigation solutions and a database that includes terrain and may include obstacles, traffic, airspace, relevant cultural features, and other data. The sensor inputs, and hence, the Synthetic Vision (SV) display is unaffected by outside weather conditions and thus, provides a clear day view regardless of the outside visibility or weather conditions.

Though there is no weather on the moon, impaired vision for the flight crew can still create technical and operational challenges or problems for lunar or planetary landings. Thrusters can create a dust cloud that can significantly reduce visibility during the critical landing phase of the flight. During Apollo, the final $100 \mathrm{ft}$ to landing was generally obscured by blowing dust, creating "a little trouble figuring out cross-range and downrange velocities". ${ }^{1}$ The absence of atmospheric haze in the lunar environment makes shadows significantly more stark, and increases the propensity for visual illusions, or removal of critical visual cues while in shadowed conditions. Landing at polar sites, in the presence of significant terrain, or in darkness, will exacerbate this problem. In addition, the attitude of the spacecraft, coupled with the window location and size, directly affects the out-the-window visibility for the crew. Unlike atmospheric aircraft that generally point in the direction they are going, spacecraft are comparatively unconstrained in attitude. Thus, a spacecraft can have its windows facing $180^{\circ}$ from the direction of travel. If the vehicle trajectory can be optimized without crew visibility being a constraint, significant fuel and weight savings may be gained. During Apollo, crew visibility and trajectory

Further author information: Steven Williams, E-mail: steven.p.williams@nasa.gov, Telephone: 17578642134 
(fuel) were traded off in the design. "The constraints placed on crew visibility by the design of the LM (Lunar Module) window and by the trajectory parameters make the viewing of the programmed landing site a major problem." 2

The Crew Vehicle Interface (CVI) group of the Integrated Intelligent Flight Deck Technologies (IIFDT) has done extensive research in the area of SVS, Enhanced Vision System (EVS) and eXternal Visibility Systems (XVS) in the aeronautics domain. SVS research has proven the efficacy of the technology to substantially enhance flight crew situation awareness, reduce workload, improve aviation safety, and increase flight technical precision. $^{3-6}$ The CVI group has been tapped to evaluate the potential applicability of Aeronautics-centric flight deck display technologies to support the United States Space Exploration Policy (USSEP). ${ }^{7}$ This paper explores the development of SVS display concepts specifically for a lunar landing vehicle. In addition, a brief overview of future work is given, specifically, that associated with evaluating the efficacy of combining SVS with XVS and EVS technology to optimize the flight deck for planetary exploration missions.

The initial design philosophy for SVS displays was to enhance the human's awareness of the vehicle state and reduce the workload of the task. It is possible to completely automate the entire landing process as evinced by Mars rovers, however the human is best at assessing and adapting to a given situation, particularly in reaction to unplanned events or failure conditions. Nonetheless, to make sound decisions, the human operator must understand the current state of the vehicle and not be task saturated. In aircraft, situation awareness is best achieved through visual cues out-the-window as well as forming a mental model based on the aircraft instruments. SVS research in aviation has showed an increase in situation awareness with reduced workload by enhancing the cockpit displays to be more intuitive to interpret. The goal is to provide enough information to keep the astronaut engaged without being task saturated so that the current situation and future states are more quickly and accurately understood. This allows for sound decision making during critical moments such as the landing phase. ${ }^{8}$

\subsection{Apollo SVS-enhanced flight deck simulations}

To investigate the potential benefits of an SVS-enhanced flight deck for spacecraft, the Apollo lunar landing mission was explored. The Apollo lunar landings were an extraordinary achievement and required the best pilots to train to become astronauts. These astronauts were tasked with navigating a new vehicle in an unknown environment with basic flight instrumentation. The flight instrumentation of the Apollo Lunar Excursion Module (LEM) consisted mainly of electro-mechanical gauges which was the typical technology for aircraft in the 1960s.

The landing phase was identified by Neil Armstrong as being the most difficult part of Apollo $11,{ }^{1}$ therefore, this project focused on the descent stage to landing. Apollo experience showed that "the constraints placed on crew visibility by the design of the LM window and by trajectory parameters make the viewing of the programmed landing site a major problem." ${ }^{2}$ The importance of pilot visibility was not only emphasized by trajectory design and window definition, but also, by conducting the Apollo landing task only at specific times and locations to provide optimal sun light on the landing site. ${ }^{1}$ The mission was designed around lighting conditions that would create shadows that would provide optimal depth perception. These optimal lighting opportunities typically lasted about a week. If the opportunity was missed, the next opportunity to land would not occur for another month.

SVS may provide three key benefits for lunar and planetary applications. First, the designer controls the SVS scene lighting, terrain coloring and virtual camera angles. Therefore, visual cues for the landing site can be created in the SVS independent of the sun-angle. Second, the information is "full field-of-regard." SV terrain presentations may be provided, conformally, in any location with respect to the vehicle or its actual window placement as required to successfully accomplish the mission. Lastly, SV provides the capability for "augmented reality" - that is, the enhancement of existing visual cues or provide conformal overlay of important vehicle state information such as forward and down velocities, altitude, and fuel remaining can be overlaid directly onto the terrain display to significantly ease pilot interpretation of the data and enhance situation awareness (Fig. 1).

A high resolution terrain map of the moon and an accurate position and orientation of the vehicle is all that is necessary in providing lunar SVS cockpit displays as 3-dimensional computer rendering of complex scenes is now becoming commonplace. High resolution terrain imagery to generate the SVS depiction should be available 

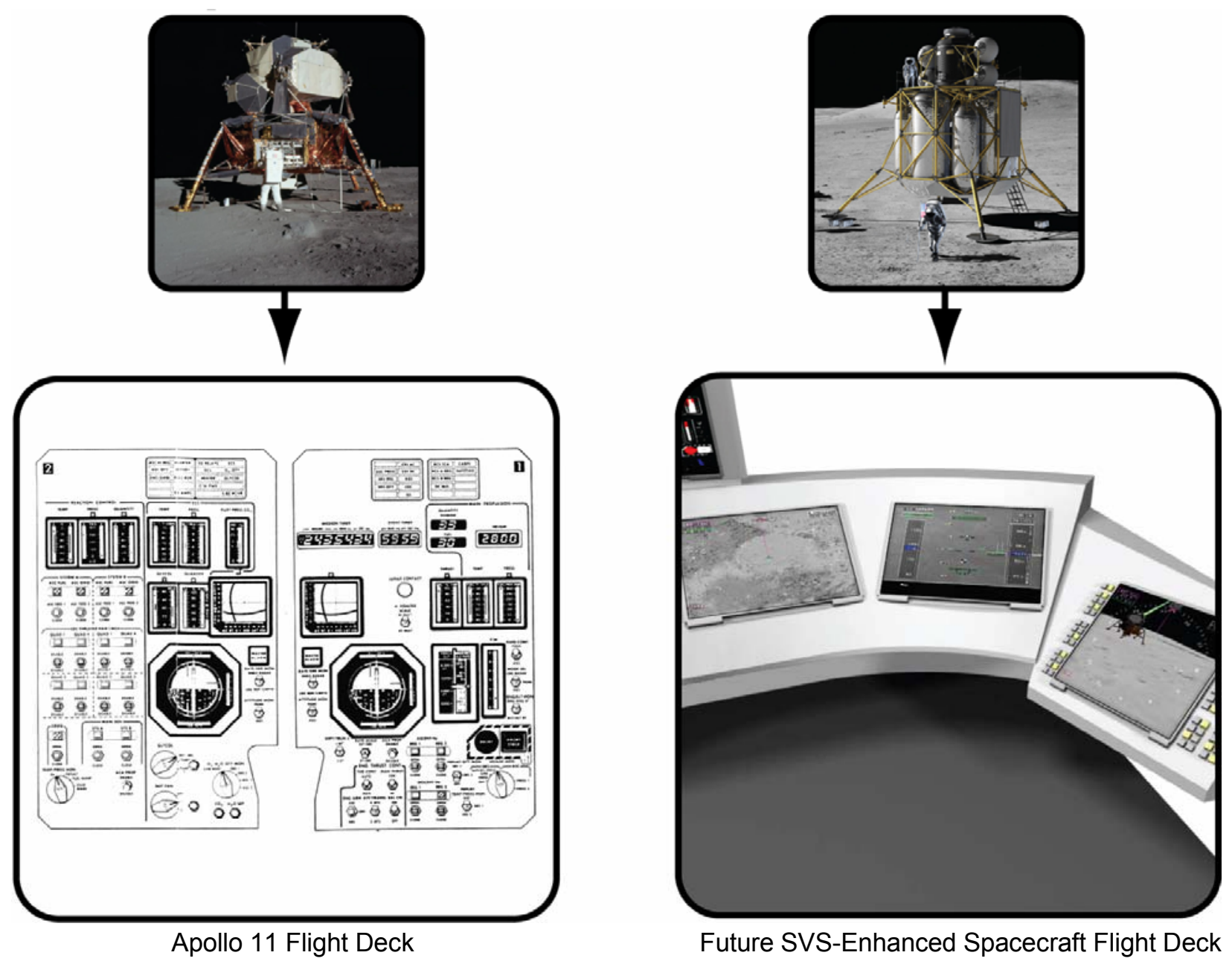

Figure 1. Concept picture showing the Apollo Lunar Module displays compared to SVS-enhanced displays. The developed Lunar SVS displays: a tactical display, a strategic display and an exocentric display.

from the Lunar Reconnaissance Orbiter (LRO) mission (see http://lunar.gsfc.nasa.gov/) and others long before manned missions to the moon begin.

Part of the research includes the role of enhanced and synthetic vision technology to support the lunar landing mission. Enhanced Vision (EV), a real-time imaging sensor, can provide two key benefits for this task. First, in lieu of a high resolution lunar terrain database, active sensors could be used to generate this data. More likely, however, on-board sensors will be used to supplement the SVS display in real-time, for instance, to ensure the landing site is free of objects not in the database (such as objects on a colonized moon). A significant aviation-domain background in research and flight experimentation/experience for optimal fusion of synthetic and enhanced vision (such as Forward Looking InfraRed (FLIR)) is being used as reference for this work. ${ }^{9,10}$ Active sensors should be capable of detecting items which are impossible to store in a static, onboard database. Further, active sensors have been developed and tested to provide additional or secondary sources for navigation, independent of the aircraft's primary navigation sources. These sensors may serve as independent integrity checks to the primary sensors or as a primary means for navigation position information when other sources are not available. NASA's research goal will be to define, from a flight deck-centric view, EV sensor roles and requirements such as field-of-view, field-of-regard, update rate, and other display parameters.

SVS provides the potential to eliminate pilot visibility as a constraint in lunar descent trajectory design, thus, enabling significant fuel savings. The descent to landing phase of the lunar approach for Apollo was designed to 
provide astronauts proper out-the-window visibility at the appropriate times. While this helps the astronauts overall situation awareness, it is not necessarily the most fuel efficient approach. Aviation-domain research and development has produced window "replacement" technologies. These aviation tested technologies will be extended to the lunar descent and landing problem.

\subsection{US Space Exploration Policy}

In 2004, NASA was directed by the President to "implement a sustained and affordable human and robotic program to explore the solar system and beyond." ${ }^{7}$ Further, NASA was directed to return humans to the moon for extended missions. The directive, known as USSEP, requires NASA to retire the space shuttle fleet and develop a new transportation system to meet the USSEP goals. In order to meet these objectives, NASA initiated the Constellation Program.

With NASA's goal to return to the moon, frequent missions to the moon will require precise landing of vehicles (possibly in the 10s of meters accuracy). It is currently planned to have several habitat modules, power generators, storage and surface mobility units. ${ }^{11}$ An advantage for using SVS-enhanced displays is that advanced precision guidance can be intuitively integrated into the displays. Therefore, the landing task will involve not only landing on suitable terrain but avoidance of human-made obstacles.

Not only do SVS displays have the potential to provide for intuitive, easily interpretable real-time cockpit displays, they may be used on-board in mission-rehearsal or during near real-time landing site re-planning tasks. The displays would enable the ability to preview the planned trajectory and directly visualize its relationship to the terrain, the landing site, and fuel status prior to flying the mission. Contingency planning and scenarios can be rehearsed and then, when executed directly on the same displays, the transfer of training is direct. The ability of the crew to handle emergency or failure conditions, particularly the aspect of fuel-/energy-management, should be critical. While this premise has not yet been tested, aviation-domain applications of this technology have been shown. ${ }^{12}$

\section{FLIGHT DECK DISPLAY DEVELOPMENT}

In the following, a baseline capability is described which has been created to support the research, development, test and evaluation of Lunar Lander flight deck concepts which employ Synthetic and Enhanced Vision. This capability is specifically designed for rapid prototyping for concept assessment and human factors research. The capability heavily relies on existing Aeronautics-centric capability, yet deviates from this focus as necessary and appropriate. The initial Lunar Lander flight deck concept, rightly or wrongly, evolved from a "typical" modern aircraft display configuration consisting of a tactical display known as a Primary Flight Display (PFD), a strategic display referred to as a Navigational Display (ND) and a auxiliary display known as an Electronic Flight Bag (EFB). Subsequent work will assess and refine these concepts to support the flight deck requirements for USSEP.

\subsection{Research concept and equipment}

The core technology development for this project was the development of lunar SVS flight deck displays. This development depended upon several essential parts: a real-time renderable model of the lunar terrain, a lunar vehicle with vehicle dynamics in a lunar environment, and development of the flight deck displays themselves. The lunar terrain model development was procured from TerraMetrics, Inc. which provided an ellipsoid moon terrain database in TerraBlocks ${ }^{\mathrm{TM}}$ format. The model development was a direct fall-out from NASA-sponsored research (under the Small Business Innovative Research (SBIR) program) to create a unique terrain database, tailored for just such an application. ${ }^{13}$

The Apollo 15 landing site was emphasized in this work because of the availability of higher resolution data and the more interesting terrain features. The delivered lunar database also contained high resolution terrain inserts for the Apollo 11 and Apollo 15 landing sites. The lunar terrain model was draped with imagery of the lunar surface to create a realistic looking and geospatially accurate database. From this core technology development NASA initiated the development and incorporation of SVS technology into specimen lunar lander flight deck displays, in simulated lunar landing scenarios. 
A rapid-prototyping simulation capability was developed in the Visual Imaging Simulator for Transport Aircraft Systems (VISTAS) laboratory. VISTAS III was a single pilot fixed-base simulator consisting of a $144^{\circ}$ by $30^{\circ}$ out-the-window visual scene, a Head Down Display (HDD), an EFB and pilot input controls. The HDD was a 21-inch LCD monitor which displayed the PFD and ND. The EFB was rendered on a touch screen, tablet PC. The pilot input controls were a typical USB gaming joystick with 12 buttons and a simple throttle control. For these simulations, the throttle control was used to tilt the virtual camera angle along the pitch axis. The flight deck development was done by four CVI personnel in approximately 3 weeks. A LEM simulation model was ported to a PC platform in VISTAS by the NASA Langley Simulation Development and Analysis Branch (SDAB).

\subsection{Lunar model}

As mentioned, one essential component to SVS cockpit displays is an onboard terrain database. Currently, the Clementine Project (1994) data is available which is on the order of 4 terrain posts (i.e., data points of latitude, longitude, and elevation) per degree. Higher resolution for global coverage terrain data were obtained for the Apollo 15 landing site. This higher resolution data is 64 terrain posts per degree which, at the lunar equator, is approximately one data point every 500 meters. Further, there is no Clementine laser altimeter data at the pole regions (above $\mathrm{N} 60^{\circ}$ and below $\mathrm{S} 60^{\circ}$ ). Stereo-pair imagery was used for generating elevation data in the polar regions. The vertical accuracy of the Clementine data was estimated at 130 meters. ${ }^{14}$ Based on aviation standards in RTCA DO-276, this resolution would not be sufficient in determining a safe landing zone solely using SV displays. Either active sensors (such a Light Detection and Ranging (LIDAR) or Forward Looking Infrared (FLIR)) or a more accurate lunar database is needed for reliance on SVS displays to touchdown. Future planned lunar missions, such as the LRO set to launch in Fall 2008, ${ }^{15}$ will provide 5-meter data for terrain and 1-meter data for imagery. This data resolution would be sufficient, based on RTCA DO-276, to support nearlyCat. III aviation operations. Testing should be conducted to evaluate what database resolution and accuracy requirements are needed to support lunar landing.

Typically, flat earth projections are used in simulations which is sufficient for localized simulations. From aviation research, this was found to be insufficient for flight operations, since flat-earth projections are inaccurate over just a few miles. Therefore, an ellipsoid model of the moon was created from the Clementine data. This database is an "on the sphere" database, therefore, there are no projection inaccuracies. Another problem with typical terrain database rendering is an effect known as "terrain popping." Typically for large databases, a computer graphics technique, known as Level of Details (LODs) switching, is used to increase the efficiency of rendering the database in real-time. As the scene changes, terrain will appear to "pop" into the scene as LOD boundaries are crossed. This popping is typically distracting to pilots flying the displays. This attribute is particularly exacerbated at orbital and sub-orbital speeds during entry and approach to landing spacecraft operations. The Terrametrics database eliminated this popping distraction.

For orbital as well as terrestrial flights, round terrain models, free of terrain popping, are essential for actual implementation into SVS-enhanced cockpit displays. In addition, the database needs to be efficiently rendered in real-time. A SBIR with TerraMetrics, Inc. developed the technology to efficiently store and render an ellipsoid database in real-time. The TerraMetrics SBIR database development focused on solving issues related to certification of such a system for commercial aviation.

An interesting benefit of SV may be as a redundant source of navigation information. For instance, the lunar model can be used in determining height above the terrain if the position of the spacecraft is known. One of the problems the Apollo $11 \mathrm{crew}$ faced was the determination of altitude. ${ }^{1}$ During the initial lunar orbits, a sextant was used to verify altitude. This was because the onboard radar system had a limited range and a fixed antenna which only worked when pointed towards the moon. Therefore, under certain altitudes and attitudes, the radar altimeter could not be used. However, with SVS, you can query the terrain database for your altitude above the moon independent of your attitude. SVS is not limited by the distance or antenna placements constraints of the radar altimeter. The fidelity of the SVS-derived altitude is dependent upon the navigation solution accuracy and the resolution of the terrain database.

Part of the research work planned in this area involves trade-studies comparing and contrasting various levels of equipage and associated sensor and database fidelities. For instance, the accuracy of the lunar terrain 
database currently used for this project would not likely be sufficient for touchdown in an actual implementation. During the high altitude portion of the approach trajectory (which is beyond the operational range of the radar altimeter) a query of the SVS terrain database using an estimate of the spacecraft's position could be of sufficient accuracy for height above the terrain. Future research could show that a less accurate database such as this one, could still provide "operational credit" for certain orbital altitudes. Therefore, trade-studies are planned to assess mission task elements and various EV and SV capabilities.

\subsection{Apollo vehicle model}

A simulation model of an Apollo vehicle was developed by SDAB. The vehicle approximated the mass properties and propulsion of the Apollo 11 Lunar Module. The simulated total weight at Powered Descent Ignition (PDI) was $33683.5 \mathrm{lb}$, including $18000 \mathrm{lb}$ of descent propellant. The descent engine had a maximum simulated thrust of $10000 \mathrm{lb}$ and an Specific Impulse (Isp) of 300. The descent engine model gimbaled for pitch and roll attitude control, with Reaction Control System (RCS) jets firing to maintain attitude errors within a specified deadband and for yaw attitude control. The modeled RCS had a total of 16 jets in 4 quadrants of 4 mutually perpendicular jets, each on the diagonal corners of the ascent stage, approximately $7.5 \mathrm{ft}$ from the ascent stage Center of Mass (CM). Each jet had $100 \mathrm{lb}$ of thrust, a Isp of 284 and a minimum firing time of 14 milliseconds. The CM of the ascent stage was approximately in the center of the RCS jet plane. The descent stage CM was approximately $2.0 \mathrm{ft}$ below the ascent stage CM, and the descent engine thrust point was $2.0 \mathrm{ft}$ below the descent stage CM. Moments of inertia were assumed to change linearly as propellants were consumed. The model assumed a spherical non-rotating moon with an inverse radius squared lunar gravity model. The model was developed to run on Linux/Unix type workstations, and was ported to a PC computer. The software was also modified to output vehicle state information to the developed Lunar SVS displays.

\subsection{Tactical display}

Tactical displays contain guidance and vehicle state information to aid the pilot in immediate navigation and control. The displays were modified from aviation-domain concepts which may not necessarily be appropriate to the lunar landing task. Research is planned to develop appropriate display concepts to support this new mission. The tactical display development was by no means "optimized" but represented a basis from which to quickly evaluate the feasibility of the lunar SVS concept.

The display initially used a velocity vector-reference format but was eventually replaced with an attitudereference format. The velocity vector display was difficult to interpret in hover mode as the velocity vector can have a large drift. Therefore, an attitude-referenced display was used with the ability to move the SVS camera position/attitude reference. This feature proved useful during the hover maneuver to view the landing spot in the SVS display independent of the vehicle attitude and trajectory. .

The tactical display was further enhanced by adding symbology. The display in Figure 2 shows a typical display on approach. A brief description of the major symbology elements are listed below:

- Tunnel - Also known as "Highway in the sky"16,17 shows a 3-dimensional representation of the intended flight trajectory. Past aeronautics research showed that this presentation of the path provided greater path performance, higher situation awareness and lower workload compared to traditional flight directors. ${ }^{18}$

- Speed and Altitude Tapes - Similar to aeronautical displays, critical information such as ground speed, vertical speed and altitude are displayed as "tapes". Tapes are useful because they provide the current data as well as the trend rates.

- Fuel Indicator - A fuel remaining symbology was developed as crew/spacecraft survival is dependent on proper fuel management. The fuel was displayed as amount remaining as a percentage as well as the amount of time (first order prediction) until the fuel is exhausted. Fuel consisted of both a text readout as well as a graphical fuel gauge.

- Pitch Ladder - A pitch ladder was provided for attitude determination. The pitch ladder is similar to attitude references in aircraft. 


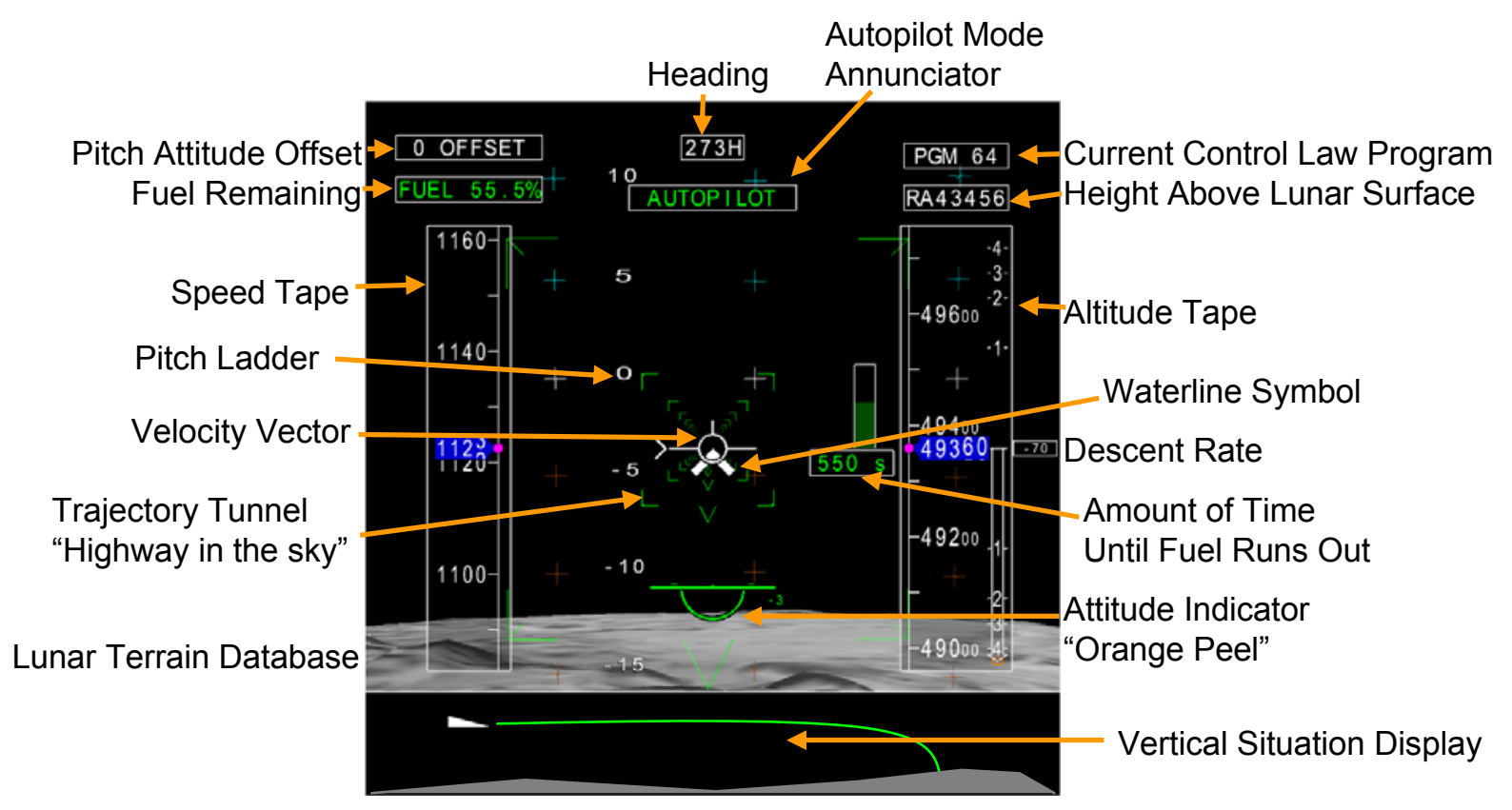

Figure 2. SVS PFD with Symbology.

- Velocity Vector - A velocity vector symbol was drawn and since it is possible that the velocity vector could be off the display screen, "ghost" symbology and logic was used to denote the direction of the velocity vector (known as a "pegged" velocity vector).

- Orange Peel - An attitude symbology, known as the "orange peel" ${ }^{19}$ was also displayed on the PFD. The orange peel was developed to aid fighter pilots to quickly determine the aircraft attitude as they tracked a target. The orange peel provides a quick attitude reference independent of the SVS camera view/PFD attitude reference.

- "Foot" Vector - The "Foot" vector is a symbol drawn $90^{\circ}$ pitched down from the water mark. The Foot vector was displayed to give the astronaut a sense of where the thruster was pointed during landing.

- Vertical Situation Display (VSD) - A preliminary VSD was developed to show the path and the terrain from a side view. The VSD provided a quick and intuitive awareness of an aircraft/spacecraft relative to the terrain.

Figure 3 shows the PFD just before touchdown. During landing, the pilot could move the virtual camera to a viewing angle directly through the floor of the spacecraft. This is denoted in Figure 3 by the text "-90 OFFSET" in the upper left corner. A reading of "0 OFFSET" indicates a typical attitude centered display for an aircraft. The Foot vector is also an indication that the display is centered about the bottom of the craft rather than the nominal aircraft position of out the nose. The nadir point $\left(-90^{\circ}\right.$ mark on the pitch ladder $)$ is depicted as a brown dot. At 500 meters, the VSD ownship symbol would transition into a lander type icon to provide an indication of pitch during hover to touchdown. At 200 meters, an orange terrain warning wedge would appear on the altitude tape. Using the Foot vector with the nadir and Landing Cone, accurate landings could be routinely made.

A touchdown zone symbology was drawn centered on the designated touchdown site and had a radius of 500 meters. Similarly, a touchdown pad was also drawn centered at the designated touchdown site and had a radius of 50 meters. Drawn at the center of the intended touchdown point was a 3-dimensional cone symbology. The cone was a green color except for the south-west quadrant which was brown. The perspective view of the cone was a rapid and intuitive way to determine the spacecraft orientation with respect to the landing point. 


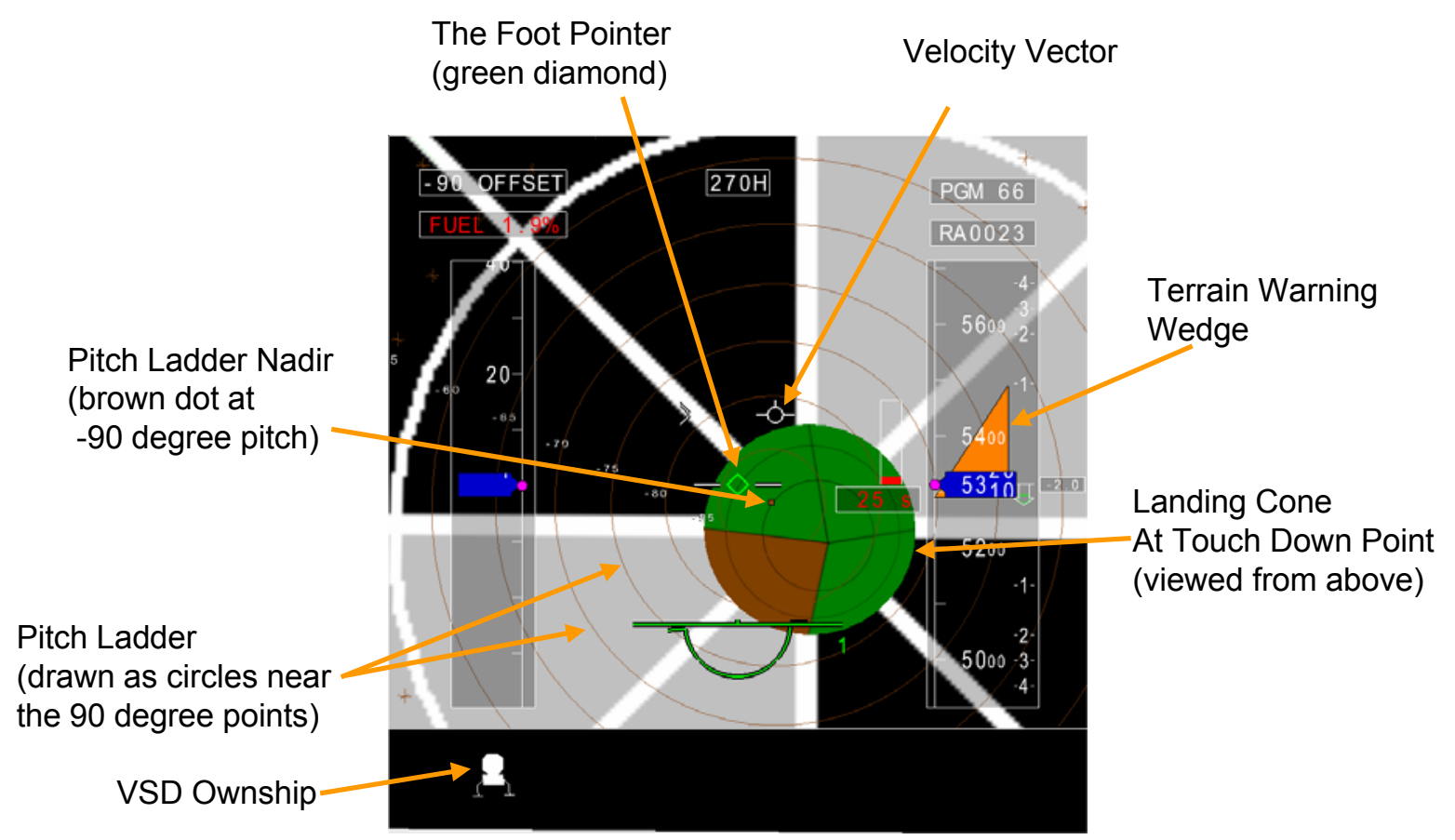

Figure 3. SVS PFD just before touchdown.

\subsection{Navigation display}

The navigation display developed was modeled after modern commerical aircraft displays. The navigation display was a two dimensional, top down view with own ship position located at the center of the display (Fig. 4). The synthetic lunar terrain was displayed under the symbology. A forward arc version of the navigation display that puts the own ship position near the lower portion of the display was considered, however a map-centered display was developed. The navigational display was pilot selectable to be oriented either track-up or heading-up.

\subsubsection{Navigational Display Symbology}

The navigation display symbology was modeled after modern aircraft displays with the exception of the zero velocity symbol. The zero velocity symbol (green circle) was a prediction of the point where the vehicle will stop moving based on its current lateral and longitudinal accelerations.

The developed symbologies for the Navigation Display were:

- Compass rose - Displayed with major tics every 10 degrees, and minor tics every 5 degrees.

- Range Ring - The diameter of the ring was half the display height and the range was indicated on the right. Range scale was pilot selectable via joystick buttons.

- Velocity Trend - The indicator was drawn from the own ship position along the ground track with segments ending at the 10, 20, and 30 second position.

- Bearing Carat - It was shown along the compass ring indicating the bearing angle to the designated touchdown target.

- Track Carat - It was shown along the compass ring indicating the current track angle.

- Cross - The center of the display represents the current own ship location.

- Zero Velocity - A circle was drawn where the vehicle was estimated to stop based on the current accelerations. 


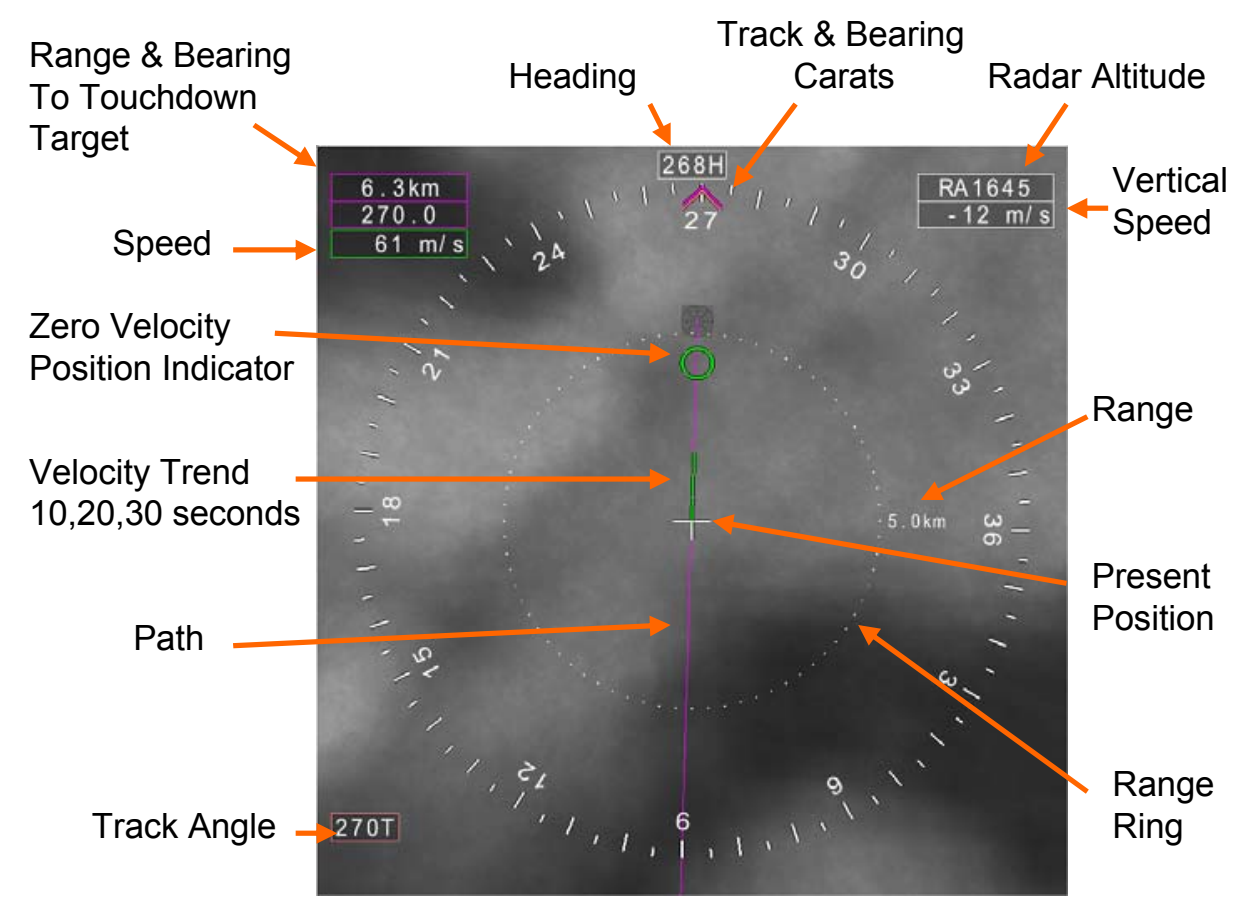

Figure 4. SVS ND with Symbology.

There are several text boxes surrounding the compass ring. The color of the box edge correlates to the symbol on the display. The magenta boxes showed the range and bearing angle to the designated touchdown site, and the magenta carat on the compass ring indicated the same angle. The green box showed the current speed and was the same color as the trend vector and zero velocity circle. Radar altitude was the height above the lunar surface and was shown in the upper right text box. Just below the radar altitude box was the vertical velocity text box. In the lower left of the display an orange box gives the off-selected orientation angle (Track angle if heading-up mode was selected, heading angle if track-up mode was selected). The orange carat indicated this angle.

\subsection{Exocentric display}

The exocentric view was a 3-dimensional representation of the lunar lander, its current velocity vector, and the surrounding synthetic terrain (Fig. 5). The exocentric view display was modeled from aircraft displays developed in previous research, known as the Mission Rehearsal Tool (MRT). ${ }^{12}$ Results from experiments with MRT showed significant situation awareness enhancement compared to paper charts. The view was from a wingman perspective (chase plane) and was positioned with respect to the rear quarter of own ship position. The view angle was manipulated such that all points of interest were within the view space; that is, the lander, and the projected touchdown location. The attitude of the lander was correctly depicted based on the real-time vehicle state data.

Much of the 2-dimensional navigation symbology was retained on this display, such as the compass ring with bearing and track angles, as well as the altitude, target data, and velocity text boxes.

\section{INITIAL EVALUTATIONS}

The three displays developed were used together for cursory real-time simulation evaluations in VISTAS. In addition, the lunar database was rendered on the out-the-window scene.

It was critical to monitor several spacecraft parameters and have a clear understanding of the spacecraft state. The descent rate must be within a safe range so as not to damage or destroy the lander on touchdown. The 


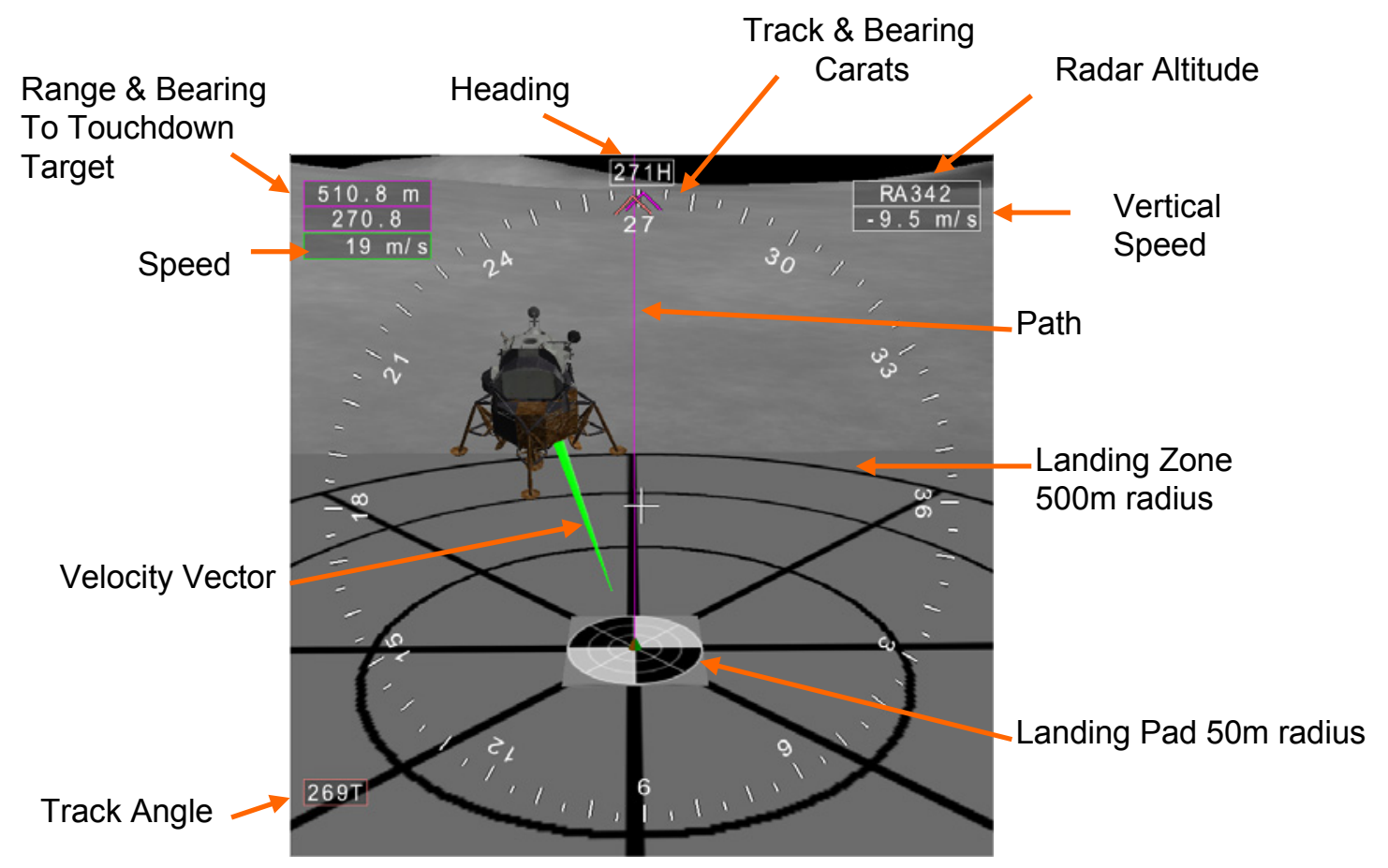

Figure 5. SVS ND exocentric view approaching the landing target zone.

proper attitude for landing must be monitored for proper landing as to not damage the landing gear. Lateral and longitudinal speeds must be zeroed to prevent skidding on touchdown. The amount of propellant must be checked frequently especially during hover. Awareness of the height above the terrain needs to be maintained. Finally, the pilot needs to ensure the landing zone is clear of obstacles and is suitable for landing.

During various flight phases, certain displays were found to be more useful. For example, during the descent, the ND with the zero velocity symbology, allowed the pilot/astronaut to set up the lunar lander in preparation for landing. During the landing phase, the PFD was utilized for tactical guidance to touchdown. The pitch offset was set to $-90^{\circ}$, thus the display was attitude centered about the bottom of the spacecraft. As the lander was descending to the surface, several symbologies were used in conjunction to provide safe and precise touchdown. Particularly, the velocity vector, nadir and touch down point were used for precise (within 1 meter) landings.

Descent awareness was provided by a readout of the descent rate as well as a descent rate arrow. Cues for descent rate were also obtained from the apparent descent in the SVS PFD. Fuel awareness was provided by 3 symbologies: percentage of fuel remaining, time until fuel is depleted and a graphical tank which drains at the current fuel consumption rate. All of the symbologies changed color as critical levels of fuel were reached. Attitude awareness was maintained by the pitch ladder and the orange peel.

To land precisely required using all of these symbologies as well as the ND velocity vector predictors. The Foot vector was an effective indication of the lander's thruster, thus its relationship with the nadir and velocity vector were used for guidance. Once these relationships were understood, accurate landings could be made with the developed displays.

The Zero velocity circle proved to be an excellent guidance cue to set pitch angle for braking after the vehicle has rolled to the upright position in the final minutes before landing. During the approach phase the vehicle was pitched back (nose up) and was slowing to the approach speed. If the pilot pitched more forward to reduce braking, then the circle moved further down range, likewise if the pilot pitched further aft (increasing braking), then the circle came closer to the own ship location. It was a simple task to center the circle on the desired touchdown spot during the approach. 


\section{CONCLUSIONS}

The core technology development for a rapid-prototyping facility to evaluate lunar and planetary vehicle flight deck designs has been successfully completed. The key component was the development of an ellipsoid lunar digital elevation model which eliminates projection errors and can be efficiently rendered in real-time. The displays developed enabled pilots to safely and accurately land the LEM after some training on the displays and space craft control. It should be duly noted that the display concepts are very rudimentary at this time and improvements would greatly improve performance and fidelity. These changes are in progress. This facility should be a valuable asset to quickly assess top-level lunar and planetary lander flight deck design issues and evaluate the potential introduction of state-of-the-art Aeronautics-centric technologies to meet NASA's Space Exploration Vision.

\section{FURTHER RESEARCH}

Near-term lunar lander work should involve all facets in the development of flight deck designs with supporting Research, Development, Test and Evaluation (RDT\&E) using this rapid-prototyping facility. In particular, trade-studies are being developed to guide pilot-in-the-loop simulation testing to provide quantitative substantiation for their outcomes. Initial emphasis will be on the use of Synthetic, Enhanced, and eXternal Visibility Systems technologies to support lunar landings. Additional display devices such as Head-Up Displays, HeadWorn Displays and other virtual displays such as the Course Optical Alignment Sight (COAS) will be included in these assessments. Finally, mission-task element analysis will be performed to evaluate the flight crew information requirements and required displays and sensors to fulfill the need. Obvious areas of research also include improved symbologies, the required accuracy of the terrain database, and the need for external sensors.

In addition, the following questions for further research are suggested by preliminary results and comments:

- Can (and if so, how can) SVS and Enhanced Vision Systems (EVS) be used to reduce the visual field of regard requirements in the external scene for the crew?

- Monitoring versus piloted task cognitive and interface issues must be addressed. What are issues associated with the monitoring task (i.e., the astronaut not controlling the vehicle, or during automatic control).

- Approaches to inhabited areas may necessitate curved segments, for instance to avoid jettisoning the descent stage into structures or personnel, in the event of landing aborts. How can SVS and associated guidance symbology best be used in curved segments, including both normal and non-normal operations.?

- Is Highway in the Sky (HITS) guidance symbology a necessary or desirable display attribute? Can HITS guidance be flown at orbital speeds, from periapsis (essentially, initiation of the approach phase)?

- How best can unlimited field-of-regard technologies, employing S/EVS capabilities be used? If virtual camera rotation is used to keep the landing site visible throughout the approach, what is the optimum strategy for rotating the virtual camera? What controls, displays, and cueing are necessary for SA and error-free usage?

- How could the virtual camera be paired with an actual sensor camera, to ensure that real-time hazards are displayed?

- What issues are associated with extending the modelled mission to planetary (Mars) approach and landing?

- Are Vertical Situation Display (VSD) requirements different for the Spacecraft planetary landing task? If so, how is this display to be used and optimized for the task? 


\section{REFERENCES}

1. D. Slayton, "Apollo: Past, present and future," in 13th Symposium Proceedings, The Society of Experimental Test Pilots, (Beverly Hills, CA), September 1969.

2. C. Hackler, J. Brickel, H. Smith, and D. Cheatham, "Lunar module pilot control considerations," Tech. Rep. NASA TN D-4131, NASA, February 1968.

3. L. Kramer, J. Arthur III, R. Bailey, and L. Prinzel III, "Flight testing an integrated synthetic vision system," in Enhanced and Synthetic Vision Proceedings of SPIE, J. G. Verly, ed., 5802, SPIE, (Bellingham, WA), 2005.

4. L. Prinzel III, L. Kramer, and J. Arthur III, "Flight test evaluation of situation awareness benefits of integrated synthetic vision system technology f or commercial aircraft," in 2005 (13th) International Symposium on Aviation Psychology, Aviation Psychology, (Oklahoma City, OK), April 2005.

5. J. J. Arthur III, L. Prinzel III, L. Kramer, R. Parrish, and R. Bailey, "Flight simulator evaluation of synthetic vision display concepts to prevent controlled flight into terrain (CFIT)," Tech. Rep. NASA/TP-2004-213008, NASA Langley Research Center, Hampton, VA, April 2004.

6. L. Kramer, L. Prinzel III, R. Bailey, J. J. Arthur III, and R. Parrish, "Flight test evaluation of synthetic vision concepts at a terrain challenged airport," Tech. Rep. NASA/TP-2004-212997, NASA Langley Research Center, Hampton, VA, February 2004.

7. NASA, "The vision for space exploration," February 2004.

8. M. Cummings, E. Wang, C. Smith, J. Marquez, M. Duppen, and S. Essama, "Conceptual human-system interface design for a lunar access vehicle," Tech. Rep. HAL2005-04, MIT for Draper Labs, Cambridge, MA, September 2005.

9. R. Bailey, L. Kramer, and L. Prinzel III, "Crew and display concepts evaluation for synthetic / enhanced vision systems," in Enhanced and Synthetic Vision Proceedings of SPIE, J. G. Verly, ed., 6226-25, SPIE, (Bellingham, WA), April 2006.

10. J. Arthur III, L. Kramer, R. Bailey, and L. Prinzel III, "Flight test comparison between enhanced vision (FLIR) and synthetic vision systems," in Enhanced and Synthetic Vision 2005, J. G. Verly, ed., 5802, SPIE, (Bellingham, WA), April 2005.

11. NASA, "Draft constellation programmatic environmental impact statement," tech. rep., NASA, Washington, DC, August 2007.

12. J. Arthur III, L. Prinzel III, S. Williams, and L. Kramer, "Synthetic vision enhanced surface operations and flight procedures rehearsal tool," in Enhanced and Synthetic Vision Proceedings of SPIE, J. G. Verly and J. J. Guell, eds., 6226, p. 62260I, SPIE, (Bellingham, WA), 2006.

13. G. A. Baxes and T. Linger, "On-the-sphere block-based 3D terrain rendering using a wavelet-encoded terrain database for SVS," Enhanced and Synthetic Vision 2006 6226(1), p. 622605, SPIE, 2006.

14. M. Rosiek, R. Kirk, and E. Howington-Kraus, "Color-coded topography and shaded relief maps of the lunar hemispheres," Lunar and Planetary Science XXXIII, 2002.

15. G. Chin, S. Brylow, M. Foote, J. Garvin, J. Kasper, J. Keller, M. Litvak, I. Mitrofanov, D. Paige, K. Raney, M. Robinson, A. Sanin, D. Smith, H. Spence, P. Spudis, S. Stern, and M. Zuber, "Lunar reconnaissance orbiter overview: The instrument suite and mission," Space Science Paper 129, pp. 391-419, April 2007.

16. R. V. Parrish, S. P. Williams, J. J. Arthur III, L. J. Kramer, R. E. Bailey, L. J. Prinzel III, and R. M. Norman, "A description of the "crow's foot" tunnel concept," Tech. Rep. NASA TM-2006-214311, NASA, June 2006.

17. J. Arthur III, L. Prinzel III, L. Kramer, and R. Bailey, "Dynamic tunnel usability study format recommendations for synthetic vision system primary flight displays," Tech. Rep. NASA TM-2006-214272, NASA, Hampton, VA, February 2006.

18. L. J. Prinzel III, J. J. Arthur III, L. J. Kramer, and R. E. Bailey, "Pathway concepts experiment for headdown synthetic vision displays," in Enhanced and Synthetic Vision Proceedings of SPIE, J. G. Verly, ed., 5424, SPIE, (Bellingham, WA), September 2004.

19. J. Jenkins, D. Sheesley, and F. Bivetto, "Helmet-mounted display symbology for enhanced trend and attitude awareness," in Helmet- and Head-Mounted Displays IX: Technologies and Applications Proceedings of SPIE, C. E. Rash and C. E. Reese, eds., 5442, SPIE, (Bellingham, WA), September 2004. 